\title{
Potent Inhibition of Human Immunodeficiency Virus Type 1 in Primary T Cells and Alveolar Macrophages by a Combination Anti-Rev Strategy Delivered in an Adeno-Associated Virus Vector
}

\author{
ROGER T. INOUYE, ${ }^{1}$ BIN DU,${ }^{1}$ DEBORAH BOLDT-HOULE, ${ }^{1}$ ANTHONY FERRANTE, ${ }^{1}$ IN-WOO PARK, \\ SCOTT M. HAMMER, ${ }^{1}$ LINGXUN DUAN ${ }^{2}$ JEROME E. GROOPMAN, ${ }^{1}$ \\ ROGER J. POMERANTZ, ${ }^{2}$ AND ERNEST F. TERWILLIGER ${ }^{1 *}$ \\ Divisions of Experimental Medicine and Hematology/Oncology and Infectious Disease, Beth Israel Deaconess Medical \\ Center and Harvard Institutes of Medicine, Boston, Massachusetts 02215, ${ }^{1}$ and Dorrance H. Hamilton Laboratories, \\ Center for Human Virology, Division of Infectious Disease, Thomas Jefferson University, \\ Philadelphia, Pennsylvania $19107^{2}$
}

Received 3 May 1996/Accepted 10 January 1997

\begin{abstract}
The rate of viral replication appears to play a pivotal role in human immunodeficiency virus type 1 (HIV-1) pathogenesis and disease progression as it outstrips the capacity of the immune system to respond. Important cellular sites for HIV-1 production include T lymphocytes and tissue macrophages. Antiviral strategies, including newer treatment modalities such as gene therapy of HIV-1-susceptible cell populations, must be capable of engendering durable inhibitory effects to HIV-1 replication in both of these primary cell types in order to be effective. Among the potential genetic targets for intervention in the HIV-1 life cycle, the Rev regulatory system, consisting of Rev and its binding site, the Rev-responsive element (RRE), stands out as particularly attractive. Rev is essential for maintaining the stability of the viral genomic RNA as well as viral mRNAs encoding key structural and regulatory proteins. Moreover, it exhibits favorable threshold kinetics, in that Rev concentrations must rise above a critical level to exert their effect. To disable Rev function, primary $T$ cells or macrophages were transduced with anti-Rev single-chain immunoglobulin (SFv) or RRE decoy genes either singly or in combination by employing adeno-associated virus vectors and then challenged with HIV-1. By directing both a protein and a nucleic acid against the normal interaction between Rev and the RRE, this genetic antiviral strategy effectively inhibited infection by either clinical or laboratory virus isolates. These results provide a framework for novel interventions to reduce virus production in the infected host.
\end{abstract}

While recent research has revealed a complex picture of viral pathogenesis, one basic tenet-that human immunodeficiency virus type 1 (HIV-1) causes quantitative and functional changes in CD4-positive immune-cell populations-remains at the heart of the disease process. The integral role of HIV-1 production and viral burden in AIDS pathogenesis and disease progression has been described at both the systemic and end-organ levels $(22,36)$. CD4-positive cells, both circulating and lymphoid-tissue-associated T lymphocytes and monocyte/macrophages, are key sites of replication and reservoirs of HIV-1.

An overall effective antiviral strategy requires that prolonged inhibitory effects on HIV-1 replication be engendered in both the circulating and solid tissue lymphocytic and macrophage populations. Although considerable progress has been made with small molecule inhibitors of key enzymes such as reverse transcriptase and protease, other genetic viral targets remain attractive sites for blockade. Genetic therapeutic strategies directed against pivotal viral replication steps offer an alternative and/or adjuvant to chemotherapeutic antiviral agents. This approach, termed intracellular immunization (1), offers the potential of altering HIV-1-susceptible immune cells

\footnotetext{
* Corresponding author. Mailing address: Division of Experimental Medicine, Beth Israel Deaconess Medical Center Harvard Institutes of Medicine, One Deaconess Rd., Boston, MA 02215. Phone: (617) 6670067. Fax: (617) 859-7010.
}

so that these processes might be aborted and the patient viral burden might be diminished.

To transduce antiviral gene cassettes into hematopoietic cells, numerous gene delivery methods have been assessed, but the primary focus has been upon traditional retrovirus-based vectors (20). Due to the limitations of retrovirus vectors for transducing nondividing cells, there has been scant data on the efficacy of genetic antivirals introduced into primary cells of the monocyte/macrophage lineage. One system, employing vectors derived from adeno-associated virus (AAV), appears to offer several advantages over other gene delivery systems, including its ability to transduce a wide range of cell types (e.g., nondividing primary cells) without apparent pathogenic effects $(27,40)$. The genome of this human parvovirus is a linear, single-stranded DNA molecule, 4,680 nucleotides in length, containing genes encoding the viral regulatory (Rep) and structural (Cap) proteins under the control of three separate promoters (33-35). The structure of its genome, including the fact that no promoter elements are contained in the viral terminal repeats, has rendered it relatively easy to derive vectors from AAV which do not include the viral promoters, as well as packaging systems which do not permit homologous recombination. Controversy remains regarding the full capabilities of gene transfer vectors derived from AAV (17), but several groups have reported success employing AAV to mediate gene delivery into either hematopoietic precursor cells or mature lymphocytes $(4,15,25,26)$. Our own findings as well as those of others have further demonstrated that AAV can be 
used to introduce foreign genes into other primary cell types, including fully differentiated lineages such as neurons $(7,13$, $21)$, with good efficiency and sustained expression of the delivered genes. Utilizing the AAV system described in this study, we have also achieved efficient transduction into circulating $\mathrm{T}$ cells and alveolar macrophages.

Among the potential targets for genetic intervention in AIDS, the Rev regulatory system holds particular promise for several reasons. First, Rev is critical for virus replication, as it is essential for appropriate posttranscriptional processing of the initial viral transcript $(5,31)$. In the absence of the Rev function, this transcript undergoes multiple splicing events which yield small messages encoding only a few viral regulatory products. Second, Rev function is absolutely dependent upon a specific interaction occurring between the Rev protein and a highly conserved mutationally intolerant Rev-responsive element (RRE), contained within the incompletely spliced viral RNAs $(2,12,32)$. Finally, unlike other key viral regulatory proteins such as Tat, the Rev system exhibits favorable threshold kinetics (30). Once the initial interaction has occurred between the core high-affinity RRE binding site and a single molecule of Rev, secondary interactions occur, resulting in the polymerization of the Rev protein along the RNA. Because of this unusual mechanism of action, a critical intracellular concentration of the Rev protein must first be achieved in order for it to be operative. Studies employing transdominant mutant Rev proteins to inhibit HIV-1 have further encouraged interest in the Rev function as a target for antivirals (38).

For this study, two anti-Rev motifs were combined to target the essential interaction between the Rev protein and its cisacting sequence, namely, an optimized RRE decoy RNA, and a synthetic single-chain immunoglobulin fragment or singlechain variable fragment ( $\mathrm{SFv}$ ) whose epitope is near the carboxyl terminus of Rev (8-10, 40). By using these two motifs together, we observed an enhancement of their overall effects. This combination gene therapy approach exhibited a marked increase in the potency of the antiviral effects over those of each of its components in both primary T-cell and macrophage populations.

\section{MATERIALS AND METHODS}

Cells. Peripheral blood mononuclear cells (PBMC) were isolated from fresh whole blood by density gradient centrifugation with Ficoll-Hypaque (Pharmacia, Piscataway, N.J.), washed with phosphate-buffered saline (PBS), and counted on a hemocytometer. CD4-positive T lymphocytes were isolated from PBMC by high-gradient magnetic cell sorting with a VarioMACS (Miltenyi Biotec Inc., Sunnyvale, Calif.), according to the manufacturer's instructions. Briefly, the PBMC were magnetically stained in PBS with CD4-positive colloidal superparamagnetic microbeads for 20 to $30 \mathrm{~min}$. After incubation, the cells were washed with a PBS-5 mM EDTA buffer supplemented with $0.5 \%$ bovine serum albumin (BSA). All procedures were performed on ice. The cell suspension was then applied to a 4\% BSA pretreated precooled high-gradient separation column saturated with the PBS-EDTA buffer and placed in the magnetic field of a permanent magnet $(0.6 \mathrm{~T})$. CD4-positive cells were collected by positive selection after depletion of the CD4-negative cells. The purity of the positive fraction was checked by flow cytometry on a Becton Dickinson FACScan with anti-CD3, -CD4, -CD14, and -CD19 fluorochromed monoclonal antibodies. By this method, the CD4-positive cell populations were determined in each case to be at least $95 \%$ CD4-positive T cells. These cells were then stimulated with $5 \mu \mathrm{g}$ of phytohemagglutinin (PHA) per $\mathrm{ml}$ and $10 \%$ T-cell stimulation factor (Collaborative Biomedical Sciences, Bedford, Mass.) for $48 \mathrm{~h}$ prior to transduction and maintained in RPMI 1640 medium with 10\% fetal bovine serum (FBS)-10\% T-stim.

Alveolar macrophages were obtained from bronchoalveolar lavages (Division of Pulmonary Medicine, Beth Israel Deaconess Medical Center, Boston, Mass.) by a previously described method (29) and were maintained in RPMI 1640 medium with $10 \%$ FBS. 293 cells, an adenovirus-transformed human embryonic kidney cell line (16), were obtained from the laboratory of R. J. Samulski and maintained in Dulbecco modified Eagle medium (DMEM) plus 10\% FBS.

Viruses. Several lymphocytotropic and monocytotropic clones or strains of HIV-1 were used to challenge primary lymphocyte and alveolar macrophage cell cultures, respectively. These included HIV-HT, which has been described previously; a highly aggressive lymphocytotropic virus raised from a recombinan provirus clone derived in E. F. Terwilliger's laboratory (6); HIVBAL, a monocytotropic strain obtained from the AIDS Reagent Repository; and HIV023-83, a low-passage clinical isolate with an in vitro zidovudine-, didanosine-, and zalcitabine-resistant phenotype and genotype obtained from the viral repository in the Research Virology section of the Beth Israel Deaconess Medical Center (24). The $50 \%$ tissue culture infective doses $\left(\right.$ TCID $\left._{50}\right)$ of lymphotropic HIV-1 stocks were determined by serial dilution series onto C8166 cells. The TCID $_{50}$ of HIVBAL was estimated with peripheral blood-derived macrophages.

A seed stock of adenovirus type 5 (Ad5) was provided by the laboratory of R. J. Samulski and grown up in 293 cells. The multiplicity of infection (MOI) was determined by serial dilution onto 293 cells.

Plasmids. The AAV vectors employed in this study were derived from a parental AAV genomic clone, pSSV9, obtained from R. J. Samulski. pAd8, containing the AAV replication and encapsidation functions flanked by terminal repeats derived from Ad5, was also supplied by R. J. Samulski. pSSV9 and pAd8 have both been described in detail elsewhere $(33,34)$. The prototype AAV vector in which the $l a c Z$ sequence was inserted instead of the different elements of the antiviral gene cassettes, AAV $\beta$-gal, has also been described previously (7).

The derivation of several anti-Rev SFv genes, including the pT7L10/H10-\#4 sequence used for these studies, by the laboratory of R. Pomerantz (Thomas Jefferson University) has been detailed elsewhere $(8,39)$. The RRE decoy motif, derived by Giver et al. (14) was appended onto the 3' end of the anti-Rev SFy coding sequence or following the cytomegalovirus immediate-early (CMV-IE) promoter by incorporating the decoy sequence into $3^{\prime}$ oligonucleotide primers and reamplifying the CMV-IE or pT7L10/H10-\#4 sequences, via PCR.

The native green fluorescent protein (GFP) gene sequence, provided in plasmid pGFP10, was a gift from M. Chalfie of Columbia University. This sequence was then modified by oligonucleotide-directed mutagenesis to provide it with a new consensus initiation sequence and red-shift its spectral properties to better mimic standard fluorescein isothiocyanate (FITC) conditions as described by Heim et al. (18).

AAV packaging. Recombinant AAV vectors were packaged, using Ad5 to provide helper functions, in combination with the trans-acting AAV factors supplied by plasmid Ad8, as previously described $(7,33,34)$. Briefly, $5 \times 10^{5} 293$ cells were plated into $100-\mathrm{mm}$ tissue culture plates and grown to 60 to $70 \%$ confluency. The cells were then infected with Ad5 at an MOI of 10 for $1 \mathrm{~h}$. Afterwards, a calcium-phosphate transfection mixture containing $6 \mu \mathrm{g}$ of Ad8, 18 $\mu \mathrm{g}$ of AAV vector plasmid, $450 \mu \mathrm{l}$ of $1 / 10$ Tris-EDTA ( $\mathrm{pH} 8.0$ ), $50 \mu \mathrm{l}$ of $2.5 \mathrm{M}$ calcium chloride, and $500 \mu \mathrm{l}$ of $2 \times$ HEPES-sodium phosphate buffer was incubated for $20 \mathrm{~min}$ at room temperature and then added to the 293 cells along with $5 \mathrm{ml}$ of Opti-MEM with $10 \%$ FBS- $120 \mu \mathrm{M}$ chloroquine. The cells were then incubated overnight at $37^{\circ} \mathrm{C}$. The following day, the cells were washed and the medium was replaced with DMEM plus $15 \%$ FBS- $1 \%$ glutamine, before incubating for an additional $48 \mathrm{~h}$ at $37^{\circ} \mathrm{C}$. The 293 cells were then removed from the plates and pelleted at $200 \times g$ for $10 \mathrm{~min}$, resuspended in $160 \mu \mathrm{l}$ of DMEM, and lysed by three freeze-thaw cycles. The lysate was then centrifuged at $360 \times g$ for $10 \mathrm{~min}$, and the supernatant was treated at $56^{\circ} \mathrm{C}$ for $30 \mathrm{~min}$ to inactivate the helper virus. The supernatant was recentrifuged at $9,000 \times g$ for $10 \mathrm{~min}$, and RNase-free DNase was added to a final concentration of $25 \mathrm{U} / \mathrm{ml}$. The stocks were incubated for $1 \mathrm{~h}$ at $37^{\circ} \mathrm{C}$ and then stored at $-70^{\circ} \mathrm{C}$ until use. A standard functional titer for each stock was estimated by serial dilution onto 293 cells, because gross differences in susceptibility to AAV were not apparent between the different cell types used in these studies. Titers were consistently on the order of $1 \times 10^{7}$ to $2 \times 10^{7} / \mathrm{ml}$.

Transient Cotransfection assay on 293 cells. AAV vector plasmid DNA $(9 \mu \mathrm{g})$ was cotransfected along with $1 \mu \mathrm{g}$ of HIV-HT proviral DNA into subconfluent 293 cells in 24-well plates, using a standard calcium-phosphate transfection protocol (Stratagene, La Jolla, Calif.). Cell supernatants were collected $48 \mathrm{~h}$ after cotransfection for HIV-1 p24 antigen assessment (enzyme-linked immunosorbent assay; Dupont). In addition to the recombinant AAV test plasmids containing antiviral expression cassettes, controls included cotransfected vector plasmids without any antiviral gene insert and the HIV-HT provirus alone.

AAV transduction. Either CD4-positive T cells or alveolar macrophages were aliquoted into 24 -well tissue culture plates at $1 \times 10^{6}$ and $5 \times 10^{5}$ cells per well, respectively. Each primary cell type was transduced with $100 \mu \mathrm{l}$ of recombinant AAV per $5 \times 10^{5}$ cells (MOI, 2 to 4 ) in serum-free DMEM and incubated for 90 min at $37^{\circ} \mathrm{C}$, after which $1 \mathrm{ml}$ of DMEM with $10 \%$ FBS was added to each well Transduction was confirmed through fluorescence-activated cell sorter (FACS) analysis of GFP-expressing cells, by immunocytochemistry for the SFv protein, or by X-Gal (5-bromo-4-chloro-3-indolyl- $\beta$-D-galactopyranoside) detection of betagalactosidase $(\beta-\mathrm{Gal})$ expression from an isogenic control AAV lacZ vector (AAV $\beta$-gal). $\mathrm{X}-\mathrm{Gal}$ assays were performed under conditions of low $\mathrm{pH}$ to inhibit any endogenous $\beta$-Gal activity.

HIV challenge of primary cells. Twenty-four hours after treatment with AAV vectors, the culture medium was removed and the cells were washed twice with serum-free RPMI 1640 medium and then inoculated with HIV-1 (MOI, about 0.1 ) in a total volume of $100 \mu \mathrm{l}$. After a 1 -h incubation at $37^{\circ} \mathrm{C}$, the inoculum was removed and the cells were washed twice with serum-free RPMI 1640 medium and then incubated at $37^{\circ} \mathrm{C}$ with $1 \mathrm{ml}$ of RPMI 1640 medium plus $10 \%$ FBS Supernatant samples were then collected, and HIV-1 p24 antigen levels were 
assessed every other day. The culture medium was replaced following each collection. HIV-1 p24 antigen levels in supernatants collected from AAV-treated cells were compared with levels in control cultures treated with an isogenic vector expressing only an indicator protein such as GFP or $\beta-G a l$. Experiments were performed a minimum of three times.

Immunofluorescent staining for SFv protein. Cell samples were washed with ice-cold PBS and then cyto-spun onto slides, with $10^{4}$ cells per slide. The cells were then fixed on the slides with $95 \%$ methanol and 5\% acetic acid for $4 \mathrm{~h}$ at $4^{\circ} \mathrm{C}$. Following three 5-min washes with PBS, the samples were then incubated with $1 \%$ BSA-PBS for at least $1 \mathrm{~h}$ at $37^{\circ} \mathrm{C}$. The primary antibody (rabbit anti-mouse IgG-h+1, lot no. A90-117A-2; Bethyl Laboratories Inc.) was then added at a 1:200 dilution in 1\% BSA-PBS, and the samples were incubated at $4^{\circ} \mathrm{C}$ overnight. The samples were then washed four times with PBS before the second antibody was added (Cy2TM goat anti-rabbit, catalog no. PA42004; Amersham Inc.) at a dilution of 1:1,000 in 1\% BSA. After being incubated for $2 \mathrm{~h}$ at $37^{\circ} \mathrm{C}$, the samples received three more washes and then were mounted under coverslips with $50 \%$ glycerol and visualized, using standard FITC conditions.

RNA extraction and cDNA synthesis. Total RNA was extracted from transduced and mock-transduced lymphocytes or alveolar macrophages by RNASTAT 60 (TEL-TEST B, Inc.) per the manufacturer's instructions and quantitated by UV spectrophotometry at $\mathrm{A}_{260}$. Five hundred nanograms of the purified RNA was subjected to reverse transcription (RT) for $1 \mathrm{~h}$ at $37^{\circ} \mathrm{C}$, using random hexamers (Perkin-Elmer) and Moloney murine leukemia virus-reverse transcriptase (Gibco-BRL) in a total volume of $25 \mu \mathrm{l}$. Following inactivation of reverse transcriptase at $95^{\circ} \mathrm{C}$ for $5 \mathrm{~min}, 12.5 \mu \mathrm{l}$ of the resulting cDNA was subjected to PCR amplification.

RT-PCR. PCR was performed to amplify a 385-bp portion of the AAV SFv/ Decoy plasmid. The primer sequences $\left(5^{\prime}\right.$ to $\left.3^{\prime}\right)$ were as follows: SFV5', GTT CGC CAG ACT CCA GAC AA, and SFV3', AGT CCA CCA TTC CGT TCT CC. Each RT-PCR was set up with approximately $250 \mathrm{ng}$ of cDNA synthesized from purified total RNA from the transduced or mock-transduced cells. The PCR was performed in a volume of $100 \mu \mathrm{l}$ with $50 \mathrm{mM} \mathrm{KCl}, 10 \mathrm{mM}$ Tris-HCl ( $\mathrm{pH} 8.3$ ), $2.5 \mathrm{mM} \mathrm{MgCl}_{2}, 200 \mu \mathrm{M}$ (four) deoxynucleoside triphosphates, $250 \mathrm{nM}$ (each) primer, and $2.5 \mathrm{U}$ of Amplitaq DNA polymerase (Perkin-Elmer Cetus, Branchburg, N.J.). Cycling conditions for the PCR were 28 cycles of $94^{\circ} \mathrm{C}$ for $30 \mathrm{~s}, 57^{\circ} \mathrm{C}$ for $30 \mathrm{~s}$, and $72^{\circ} \mathrm{C}$ for $60 \mathrm{~s}$, followed by a $7-\min 72^{\circ} \mathrm{C}$ extension in a Perkin-Elmer 9600 thermal cycler.

Liquid hybridization-gel retardation analysis. Following amplification, $30 \mu \mathrm{l}$ of the PCR product was denatured and subjected to liquid hybridization $(10 \mathrm{~min}$ at $\left.55^{\circ} \mathrm{C}\right)(11)$ with $250,000 \mathrm{cpm}$ of an internal ${ }^{32} \mathrm{P}$-end-labelled oligonucleotide probe specific for the SFv/Decoy region. The probe sequence was CGA TTC ACC ATC TCC AGA GAC AAT CCC AAG, which corresponds to a region immediately $5^{\prime}$ to the location of the $3^{\prime}$ SFV primer. Hybridization products were subjected to gel retardation analyses through an $8 \%$ polyacrylamide Tris-borateEDTA gel and visualized overnight by autoradiography.

\section{RESULTS}

Construction of AAV vector plasmids. The structures of the recombinant AAV vectors, including their relevant restriction sites and vector constituents, are outlined in Fig. 1. Briefly, vector plasmids were created by substituting the antiviral or other gene cassettes in place of the native AAV coding sequences, which are located between the two inverted terminal repeats in pSSV9 and flanked by $X b a I$ sites. In each case, gene expression was driven by the CMV-IE promoter (Stratagene). Polyadenylation was provided by a 550-bp simian virus 40 segment encompassing the early polyadenylation sequence and small $t$ intron. The derivation and properties of AAV $\beta$-gal, the prototype AAV lac $Z$ vector in this series, have already been described (7).

pT7L10/H10-\#4 encodes an anti-Rev SFv whose epitope is within the $\mathrm{C}$ terminus of $\operatorname{Rev}(8,39)$. The RRE decoy used was derived from an optimized version of the minimal binding domain of the RRE stem-loop IIB structure described by Giver et al. (14), which has been shown in binding studies to exhibit up to a 16-fold-higher affinity for Rev than the wildtype RRE. This decoy, because it incorporates only the minimal binding domain, does not itself render gene expression from mRNAs containing the sequence dependent upon the presence of the Rev protein. Because cellular factors are involved in Rev function, use of a mimic to the minimum binding domain of the RRE, rather than to the entire element, should also minimize the risk of deleterious interactions occurring between the decoy and the host cell. This decoy was appended
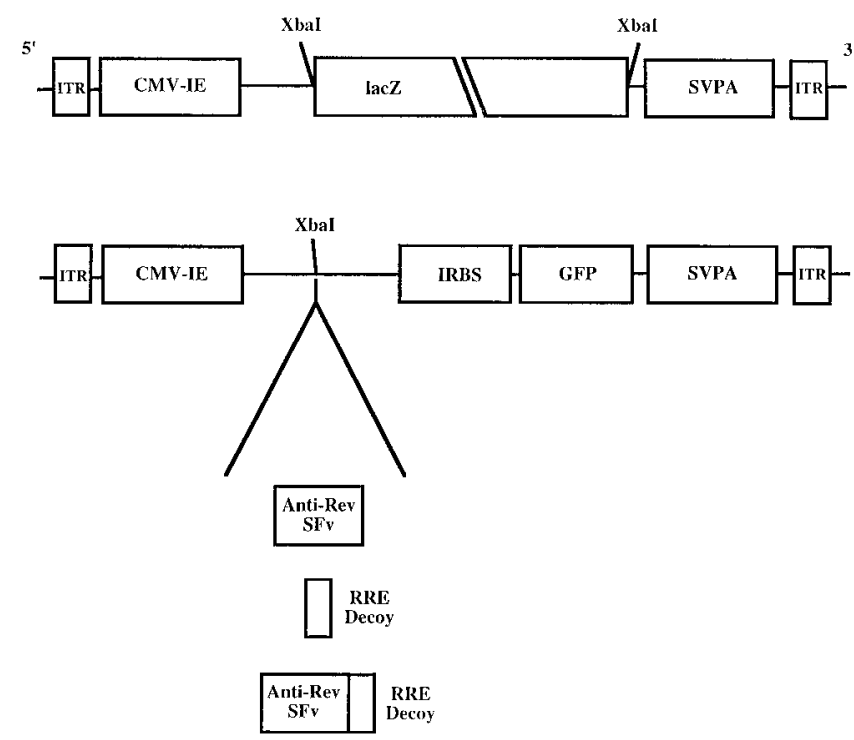

FIG. 1. Structures of AAV vectors. AAV vector plasmids were constructed by substituting antiviral gene cassettes in place of the native AAV coding sequences located between the inverted terminal repeats (ITRs) in pSSV9. Gene expression was driven in each case by the CMV-IE promoter (Stratagene). Polyadenylation was provided by a simian virus 40 sequence (SVPA) comprising the early region poly(A) signal and small $t$ intron. An IRBS from the encephalomyocarditis virus, followed immediately by the gene for GFP, was also inserted.

either to the coding sequences of pT7L10/H10-\#4 or directly following the CMV-IE sequence, by PCR (see Materials and Methods for details).

Because of the packaging capabilities of AAV (up to about $5.1 \mathrm{~kb})$ and the relatively small size of the gene cassettes involved, each antiviral vector constructed was also able to accommodate an additional marker gene. An internal ribosome binding site (IRBS) from encephalomyocarditis virus was employed to permit the efficient translation of both the SFv gene and the second indicator gene from the same mRNA (19). The gene for GFP (3), modified as described by Heim et al. (18) to shift the native protein's absorption and emission peaks closer to standard FITC conditions, was incorporated downstream from the IRBS to assist in the confirmation of successful vector transduction by fluorescent cell cytometric techniques and to eventually enable sorting of live transduced cell populations.

Transient Cotransfection assays. As an initial, rapid screen for efficacy against HIV-1 by the recombinant anti-Rev AAV, unpackaged AAV vector plasmids were transfected together with an HIV-1 provirus plasmid DNA (HIV-HT) into 293 cells. HIV-HT is a highly replication-competent, T-cell-tropic HIV-1 laboratory clone with an intact viral genome (6). 293 cells, an adherent human embryonic cell line, were chosen for their relative permissiveness to efficient transfection and the fact that they do not express CD4. As a result, virus infection could not spread within the transfected cultures. Antiviral effects of the AAV gene cassettes were analyzed by assaying HIV-1 p24 antigen levels in supernatants harvested $48 \mathrm{~h}$ after cotransfection.

293 cells cotransfected with test plasmids, individually expressing the anti-Rev SFv or the RRE decoy, reproducibly exhibited significantly (5- to 15 -fold) lower HIV-1 p24 antigen levels when compared with those in cultures cotransfected with an isogenic control AAV plasmid containing the SFv sequence inserted in the antisense orientation (Fig. 2). Expression of the 


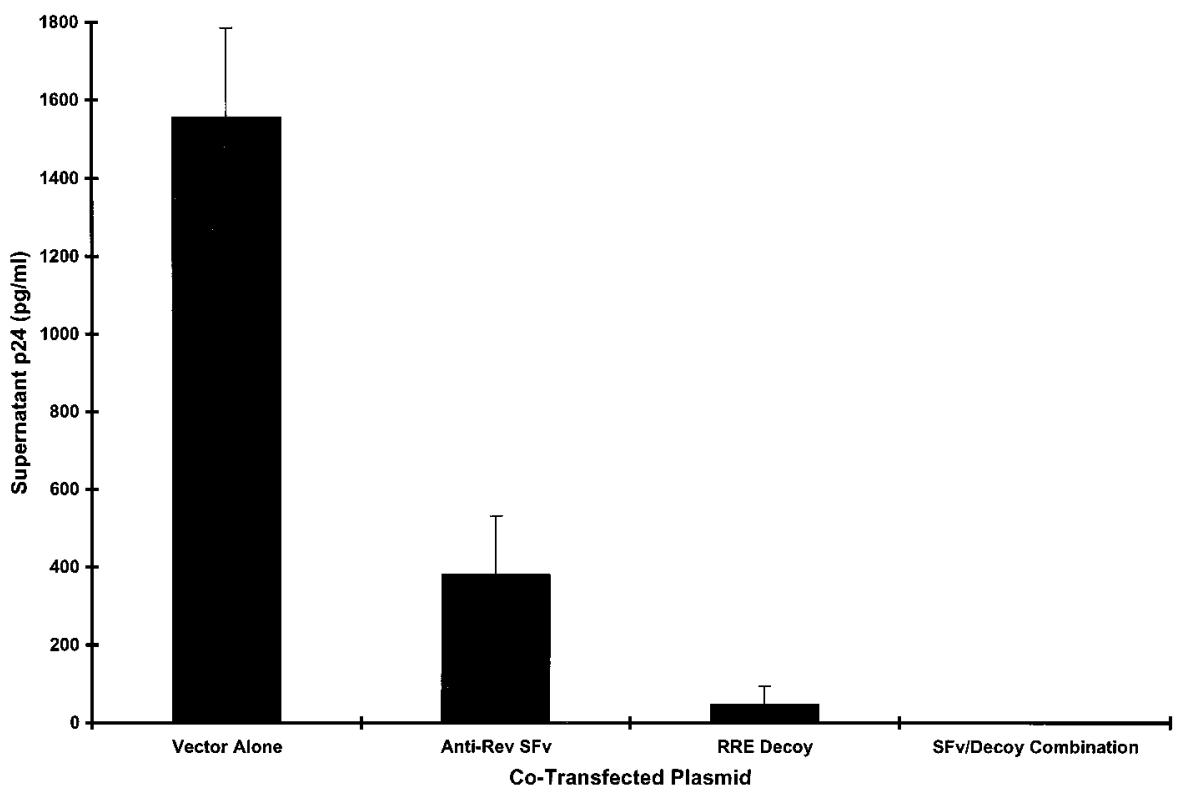

FIG. 2. Cotransfection of 293 cells with recombinant AAV plasmids and an HIV-1 provirus. As a preliminary index of efficacy, adherent 293 cells were transfected with AAV vector plasmids expressing different antivirals, together with the HIV-HT provirus, under the conditions described in Materials and Methods. Cotransfection with an isogenic plasmid carrying the SFv sequence in the antisense orientation was included as a negative control. Culture supernatants were collected $48 \mathrm{~h}$ posttransfection and assayed for HIV-1 p24 antigen levels. The data shown depicts the statistical means together with their standard deviations from two independent experiments, each performed in duplicate $(n=4)$.

anti-Rev SFv in the cells was confirmed by immunocytochemical staining (data not shown). Moreover, the vector combining the anti-Rev SFv with the RRE decoy yielded more pronounced inhibitory effects than either gene expressed alone, reflected by a further reduction of at least 10-fold in HIV-1 p24 levels.

Challenge of AAV-treated primary CD4-positive $\mathrm{T}$ cells with HIV-1. Encouraged by these preliminary findings, we next packaged vector plasmids expressing the SFv alone, the RRE decoy alone, or both together into AAV virions (see Materials and Methods for details). An isogenic vector expressing only GFP was included as a negative control, along with AAV $\beta$-gal, the prototype $l a c Z$ vector, to provide an additional easy measure of transduction efficiency. This set of vectors was then used to transduce fresh, purified CD4-positive T cells 2 days after PHA stimulation. X-Gal staining performed 3 days later on cultures transduced with the lac $Z$ vector indicated that greater than $95 \%$ of the cells had been successfully transduced (Fig. 3A and B). GFP fluorescence could be demonstrated by FACS analysis in about half the proportion of cells indicated to be successfully transduced by the $l a c Z$ vector. This latter finding was consistent regardless of cell type, whether the GFP gene was in the first or second position in the vector, or whether the DNA was delivered by transfection rather than mediated by AAV. These results indicated a diminished efficiency of detection of GFP by FACS analysis compared with the $\mathrm{X}-\mathrm{Gal}$ assay for $\beta-\mathrm{Gal}$, rather than a restricted reinitiation of translation from the IRBS sequence. Lymphocyte samples transduced with the AAV SFv/Decoy vector were also fixed and subjected to immunofluorescent staining employing a commercially available rabbit anti-mouse immunoglobulin $\mathrm{G}$ reactive with the $\mathrm{SFv}$ (39) to further demonstrate expression from the delivered transgene (Fig. 4).

Three days following AAV treatment, the cells were challenged for $1 \mathrm{~h}$ with a virus stock derived from the HIV-HT provirus and monitored for signs of subsequent virus infection.
An MOI of 0.1 was selected to approximate a physiological level of challenge. Results of a typical experiment are shown in Fig. 5. Pretreatment with AAV vectors expressing either the SFv or the RRE decoy resulted in intermediate levels of inhibition of HIV-1 production. The strongest inhibitory effects were consistently conferred by the AAV vector delivering both the anti-Rev SFv and RRE decoy genes. The absence of viral inhibition in cultures exposed to a control AAV GFP vector further showed that this suppression was not the result of treatment with an AAV vector per se. In multiple independent experiments, the difference in efficacy (up to $2 \operatorname{logs}$ ) between vectors expressing the single antivirals and the vector conferring the combination was more dramatic than that which had been observed earlier with transfected 293 cultures (compare Fig. 2 and 5). In some experiments, no HIV-1 p24 was detectable for the lifetime of the cells (2 to 3 weeks) in cultures pretreated with the double vector, although the cultures turned weakly positive for HIV-1 proviral sequences following virus challenge as determined by PCR (data not shown).

The experiments conducted to this point had made use of a laboratory clone of HIV-1 for challenge purposes. Such strains of the virus have not always been reliable predictors of the responses of unadapted clinical isolates of the virus to challenge with antivirals. For the next set of experiments, sorted CD4-positive T cells were transduced either with the best antiviral AAV vector expressing the SFv/Decoy combination or with the isogenic control vector expressing GFP alone. These cells were then challenged with a well-characterized multidrugresistant clinical isolate, HIV023-83 (24). Interference of the combination SFv/Decoy vector with the replication of the clinical strain appeared to be as dramatic as in the case of the cloned lab virus. Once again, viral expression was dramatically inhibited, by greater than 3 logs, reflected by HIV-1 p24 antigen levels which were diminished to very low or undetectable levels throughout the duration of the cultures (Fig. 6). 

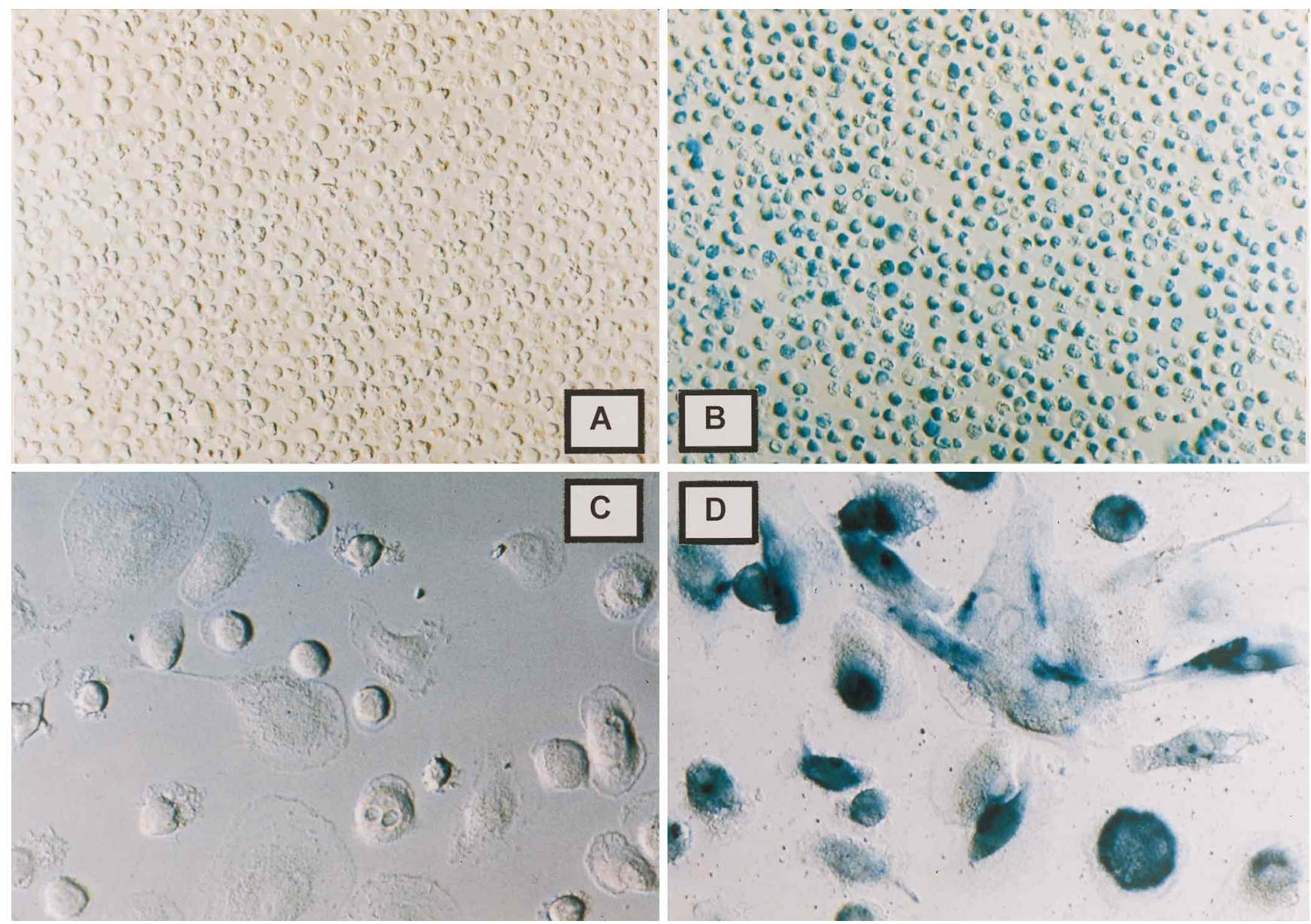

FIG. 3. $\beta$-Gal expression in primary cells transduced with an AAV lacZ vector. Purified CD4-positive T cells (B) or alveolar macrophages (D) were exposed to an AAV $\beta$-gal vector under the conditions described in Materials and Methods. Three days posttransduction, the cells were collected and subjected to a standard X-Gal staining protocol to detect $\beta$-Gal activity. Untreated control cultures (A and C) were stained in parallel as negative controls.

Challenge of transduced alveolar macrophages with HIVBAL. Despite their important roles in the pathogenesis of HIV-1 disease, examination of the utility of genetic strategies against the virus in monocyte/macrophage populations has been inhibited by the relative resistance of these cell types to efficient transduction with traditional murine retrovirus vectors. However, when AAV vectors were employed, efficiencies of transduction into mature, nondividing alveolar macrophages isolated from bronchoalveolar lavage samples were routinely in excess of $90 \%$, as reflected by the activity of the AAV $\beta$-gal vector (Fig. 3C and D). Expression of the indicator gene persisted for the lifetime of the cultures, approximately 4 to 6 weeks. This success was achieved despite the presence of only minimal levels of DNA synthesis in these cultures, confirmed by a nonradioactive DNA incorporation assay (data not shown) and fully consistent with the nondividing status of the cells.

To confirm expression of the SFv/Decoy transgene and the presence of the RRE decoy sequence in this generally transduction-resistant cell type, RT-PCR was used to specifically amplify a segment of the mRNA transcribed from the SFv/ Decoy gene cassette in alveolar macrophages following treatment with this AAV vector (Fig. 7). Parallel amplifications were performed on both AAV-treated and untreated lymphocytes. The amplified segment incorporates both the $3^{\prime}$ end of the SFv coding sequence and the 5' portion of the RRE decoy, demonstrating the presence of the mRNA and the decoy in treated cells of both types. No amplification product was observed in control lymphocytes or macrophages not exposed to the AAV SFv/Decoy vector.

Unlike virus infection of activated lymphocytes, exposure of mature macrophages to HIV-1 more typically results in a sustained low-level infection. Nevertheless, transduction of alveolar macrophages with selected anti-Rev recombinant AAV still yielded significant protective effects against subsequent challenge with HIVBAL, a strongly monocytotropic laboratory strain. These protective effects paralleled those seen with primary $\mathrm{T}$ cells, including the relative efficacies of the single versus double vectors. Supernatant HIV-1 p24 antigen levels were suppressed by each of the anti-Rev vectors but were most dramatically (15- to 20-fold) suppressed by the AAV vector combining the anti-Rev SFv and RRE decoy genes. Results from a representative trial are illustrated in Fig. 8. No virus suppression was induced by an isogenic vector expressing only the lac $Z$ gene sequence.

\section{DISCUSSION}

We have confirmed the feasibility of suppressing HIV-1 replication in two important cellular reservoirs of virus-termi- 
A

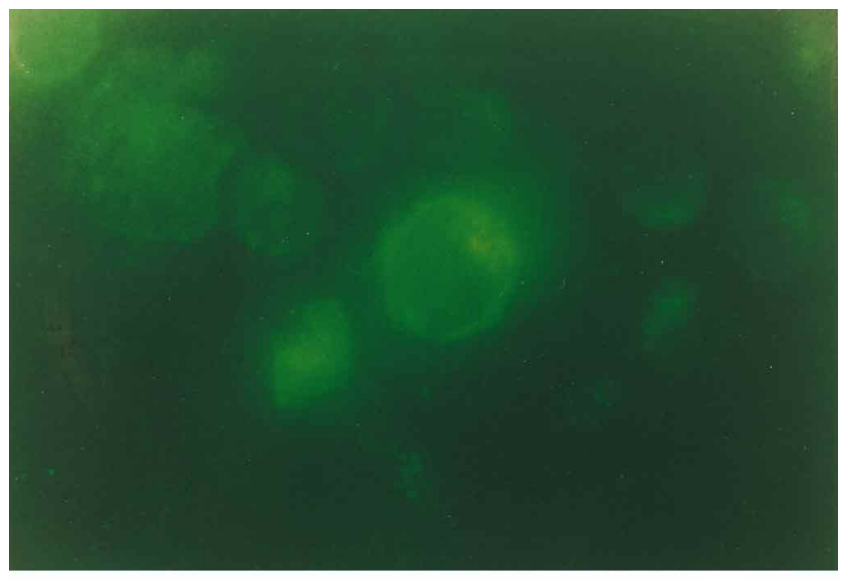

B

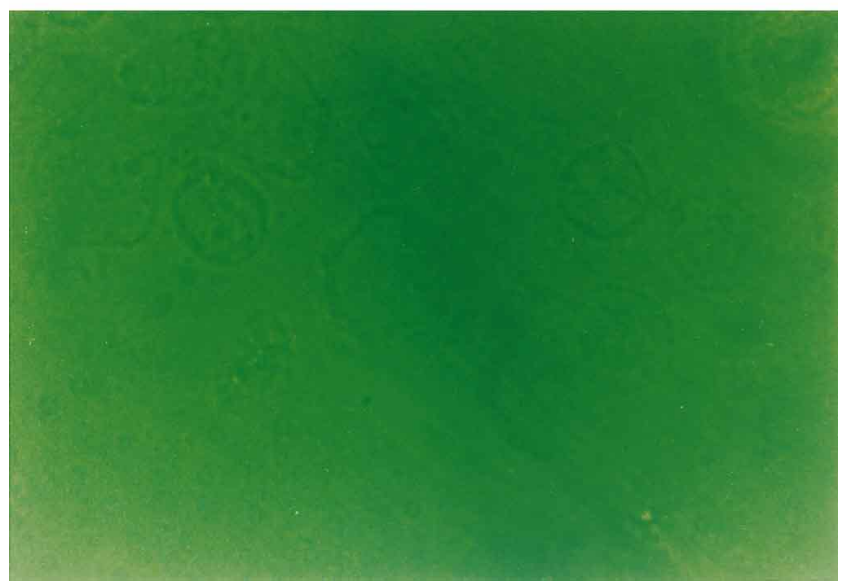

FIG. 4. Visualization of SFv protein in lymphocytes following transduction with the AAV SFv/Decoy vector. Lymphocytes were fixed 4 days posttransduction with the AAV SFv/Decoy vector and stained as described in Materials and Methods. (A) Cells visualized under FITC conditions; (B) same field under normal light.

nally differentiated tissue macrophages, specifically alveolar macrophages, and circulating $\mathrm{T}$ lymphocytes-through the transduction of combination anti-HIV AAV vectors specifically targeting the Rev regulatory system. Treatment with an anti-Rev AAV encoding an anti-Rev SFv, together with an optimized Rev binding site (RRE) decoy, strongly inhibited the replication of multiple HIV-1 isolates, including a multidrug-resistant clinical isolate, resulting in up to a 3-log reduction in viral replication as reflected by HIV-1 p24 antigen levels (Fig. 5 and 6). Cultures exhibiting strong resistance still became weakly PCR positive for HIV-1 proviral sequences following virus challenge. This outcome was consistent with the expectation that the block to virus replication provided by the delivered genes was in the postintegration phase of the viral life cycle, but virus replication was severely impeded. No suppression of HIV-1 was produced following exposure to AAV vectors harboring unrelated gene sequences such as lac $Z$. While antiretroviral effects of wild-type AAV have been reported, these effects have been localized to the rep gene cassette of the virus, which has been deleted in our vectors together with the other AAV coding sequences.

Synergistic HIV-1 inhibition was obtained by combining the

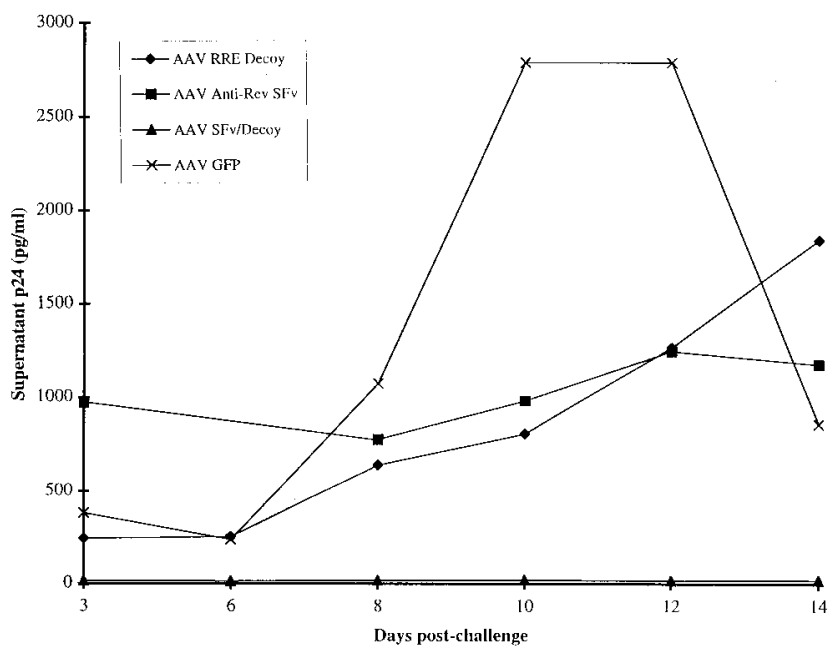

FIG. 5. Resistance of CD4-positive T lymphocytes to HIV-1 challenge following transduction with Rev antivirals. Purified CD4-positive T cells were PHA activated and then treated with one of the set of AAV vectors diagrammed in Fig. 1. Pretreatment with an isogenic vector expressing only the gene for GFP was included as a negative control. Three days later, the cells were exposed to HIV-HT (MOI, 0.1), and supernatant p24 production was then monitored to assess subsequent virus production over time.

anti-Rev SFv and the RRE decoy in a single vector. The stronger block to virus replication generated by this combination, as opposed to that seen with either antiviral alone, was particularly dramatic in the T-cell experiments. In these cultures, where the levels of virus replication are relatively high in the absence of treatment with AAV vectors, any degree of virus breakthrough became magnified over time as the HIV-1 spread through the cultures. However, the improved potency of the SFv/Decoy combination was still manifest in the context of infected alveolar macrophages, which exhibit relatively low levels of HIV-1 replication, and even in transfection experiments into CD4-negative 293 cells, where no virus spread could occur. By combining the SFv and decoy motifs, the

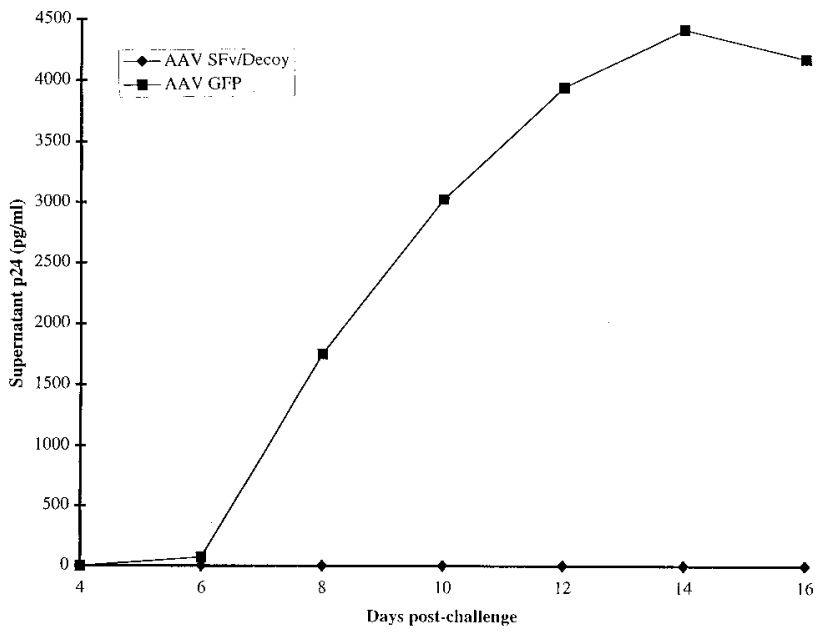

FIG. 6. Protection of AAV-treated CD4-positive T cells against challenge with a multidrug-resistant primary virus isolate. Purified, PHA-activated T cells were pretreated with either the dual AAV SFv/Decoy vector or the vector expressing only GFP as a negative control. Three days posttreatment, the cultures were exposed to HIV023-83 (MOI, 0.1), and virus production was monitored over time. 


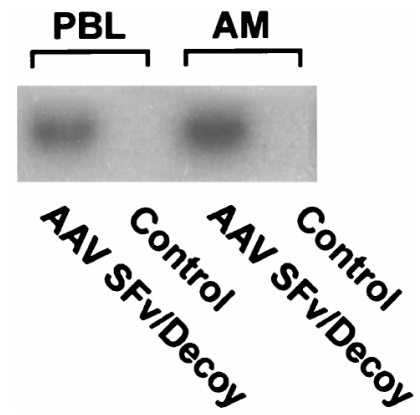

FIG. 7. RT-PCR analyses of AAV SFv/Decoy transduction in lymphocytes and alveolar macrophages. Vector gene sequences in RNA purified from transduced lymphocytes or alveolar macrophages were detected by RT-PCR amplification of a 385-bp fragment of the AAV SFv/Decoy vector. In each case, RNA from cells of the same type receiving no vector was processed in parallel as a negative control. Amplification products were visualized by liquid hybridizationgel retardation analyses, using a ${ }^{32} \mathrm{P}$-labelled vector-specific probe, as described in Materials and Methods. Each lane represents the product amplified from 250 ng of cellular RNA. PBL, peripheral blood lymphocytes; AM, alveolar macrophages.

normal Rev-RRE interaction was interfered with by two very different strategies converging on a single target protein. The $\mathrm{SFv}$ is localized to the cytoplasm and may exert its antiviral effect by shortening the half-life of the Rev protein as well as by shifting its subcellular distribution away from the nucleus $(23,39)$. The RRE decoy serves to further diminish the number of Rev molecules among the fraction remaining in the nucleus which are available to bind to native RRE sequences. The potency of this effect is consistent with what is known of Rev protein physiology; specifically, that Rev-mediated posttranscriptional processing depends upon Rev concentrations attaining a threshold level, below which its essential replication function is lost (30). Multiple anti-Rev agents may therefore be designed to work in concert to maintain effective Rev concentrations below this critical point. This goal appears more at-

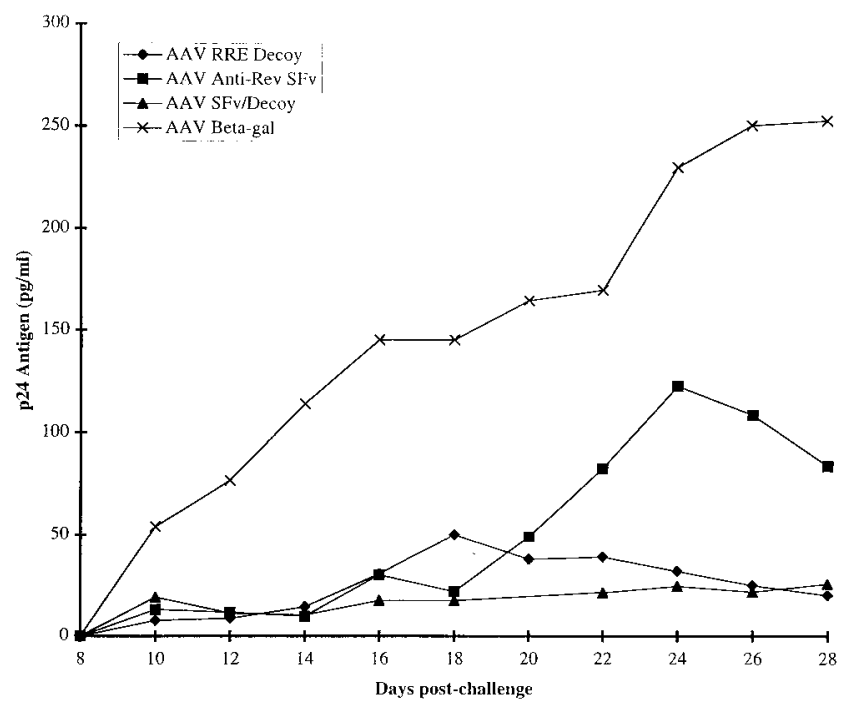

FIG. 8. Protection of AAV-treated primary alveolar macrophages agains HIV-1 challenge. Adherent cultures of primary alveolar macrophages derived from broncholavage specimens were treated with the set of antiviral AAV vectors and then exposed 3 days later to HIVBAL (MOI, 0.1). Pretreatment with a vector expressing only $l a c Z$ was included as a negative control. Culture supernatant HIV-1 p24 antigen levels were monitored to assess production of HIV-1 over time from each culture. tainable than strategies which depend for their efficacy upon successful stoichiometric interference with virtually every molecule of a viral product.

With respect to the AAV vector delivery system employed in these studies, controversy remains regarding its full capabilities, particularly concerning the form in which the vector DNA persists in the target cells and the extent to which integration occurs into the host cell genome (17). However, even if AAVdelivered DNA were maintained largely as an episome exhibiting long-term stability, the system would still have utility for some clinical applications. Of greater importance, based on our data, one would not expect the efficacy of this antiviral combination to be limited by the delivery system used. As other promising vector systems are developed (28), efficient delivery of the constructs studied in this work will be assessed. Conversely, new antiviral strategies can be evaluated with equal speed and ease in key primary cell types by taking advantage of the unique properties of AAV.

It is also noteworthy that, in this strategy, both a protein and a nucleic acid were employed together to target two distinct domains in a single key viral protein. The RRE binding domain targeted by the decoy is localized in the amino-terminal half of Rev, while the epitope for the SFv is near the carboxyl end of the protein (39). Such a dual attack should minimize opportunities for the virus to evade this genetic strategy by mutation, as has so often occurred in response to chemotherapeutics. Acquired resistance would presumably require separate changes in two different regions of Rev. In preliminary studies, we have thus far been unable to select an HIV-1 variant exhibiting resistance to the combination strategy by serial passage through $\mathrm{T}$ cells expressing the antivirals (unpublished observation). Based on what is being observed in antiretrovirus chemotherapeutics, with combination therapies employing both reverse transcriptase and protease inhibitors (37), further improvements in the genetic strategy may be gained by combining these anti-Rev elements with divergently targeted genetic antiviral motifs which interfere with more proximal or distal steps of viral replication or by combining these genetic antivirals with conventional chemotherapeutics.

\section{ACKNOWLEDGMENTS}

This research was funded, in part, by grants to E. F. Terwilliger from the American Foundation for AIDS Research, Concerned Parents for AIDS Research, and NHLBI (grant no. 2PO1 HL43510) and by USPHS grants AI36552, AI38666, AI33810, and NS30916 to R. J. Pomerantz. R. Inouye is the recipient of a National Foundation for Infectious Diseases/Eli Lilly Fellowship in Infectious Diseases.

We thank R. J. Samulski and X. Xiao for their generous provision of AAV vector starting reagents and protocols, Henry Koziel of the Beth Israel Deaconess Medical Center Pulmonary Laboratory for providing the alveolar cell preparations used in the macrophage experiments, Martin Chalfie for providing the GFP gene, Jacqueline Gillis and Patricia Price for expert technical assistance, Janet Delahanty for proofreading and preparation of the manuscript, and Tee Trac for typing assistance.

\section{REFERENCES}

1. Baltimore, D. 1988. Intracellular immunization. Nature 335:395-396.

2. Bartel, D. P., M. Zapp, M. R. Green, and J. W. Szostak. 1991. HIV-1 Rev regulation involves recognition of non-Watson-Crick base pairs in viral RNA. Cell 67:529-536.

3. Chalfie, M., Y. Tu, G. Euskirchen, W. W. Ward, and D. C. Prasher. 1994. Green fluorescent protein as a marker for gene expression. Science 263:802805.

4. Chatterjee, S., P. R. Johnson, and K. K. Wong, Jr. 1992. Dual target inhibition of HIV-1 in vitro by means of an AAV anti-sense vector. Science 258:1485-1488.

5. Cullen, B. 1992. Mechanism of action of regulatory proteins encoded by complex retroviruses. Microbiol. Rev. 56:375-394. 
6. Du, B., A. Wolf, and E. Terwilliger. 1993. Changes in the host range and growth potential of an HIV-1 clone are conferred by the vpu gene. Virology 195:260-264.

7. Du, B., P. Wu, D. M. Boldt-Houle, and E. Terwilliger. 1996. Efficient transduction of human neurons with an adeno-associated virus vector. Gene Ther. 3: 254-261.

8. Duan, L., O. Bagasra, M. Laughlin, J. Oakes, and R. Pomerantz. 1994. Potent inhibition of human immunodeficiency virus replication by an intracellular anti-Rev single-chain antibody. Proc. Natl. Acad. Sci. USA 91:50755079 .

9. Duan, L., H. Zhang, J. W. Oakes, O. Bagasra, and R. J. Pomerantz. 1994. Molecular and virological effects of intracellular anti-rev single-chain variable fragments on the expression of various human immunodeficiency virus-1 strains. Hum. Gene Ther. 5:1315-1324.

10. Duan, L., M. Zhu, O. Bagasra, and R. J. Pomerantz. 1995. Intracellular immunization against HIV-1 infection of human T lymphocytes: utility of anti-rev single-chain variable fragments. Hum. Gene Ther. 6:1561-1573.

11. Ehrlich, G. D., S. Greenberg, and M. A. Abbott. 1990. Detection of human T-cell lymphoma/leukemia viruses, p. 325-336. In M. A. Innis, D. H. Gelfland, J. T. Srinsky, and T. J. White (ed.), PCR protocols: a guide to methods and applications. Academic Press, San Diego, Calif.

12. Fischer, U., J. Huber, W. C. Boelens, I. W. Mattaj, and R. Luhrmann. 1995 The HIV-1 Rev activation domain is a nuclear export signal that accesses an export pathway used by specific cellular RNAs. Cell 82:475-483.

13. Flotte, T. R., S. A. Afione, C. Conrad, S. A. McGrath, R. Solow, H. Oka, P. L. Zeitlin, W. B. Guggino, and B. J. Carter. 1993. Stable in vivo expression of the cystic fibrosis transmembrane conductance regulator with an adenoassociated virus vector. Proc. Natl. Acad. Sci. USA 90:10613-10617.

14. Giver, L., D. Bartel, M. Zapp, A. Pawul, M. Green, and A. D. Ellington. 1993. Selective optimization of the Rev-binding element of HIV-1. Nucleic Acids Res. 21:5509-5516.

15. Goodman, S., X. Xiao, R. E. Donahue, A. Moulton, J. Miller, C. Walsh, N. S. Young, R. J. Samulski, and A. W. Nienhuis. 1994. Recombinant AAVmediated gene transfer into hematopoietic progenitor cells. Blood 84:14921500 .

16. Graham, F. L., J. Smiley, W. C. Russell, and R. Naiva. 1977. Characteristics of a human cell line transformed by DNA from human adenovirus type 5 . J. Gen. Virol. 36:59-72.

17. Halbert, C. L., I. E. Alexander, G. M. Wolgamot, and A. D. Miller. 1995 Adeno-associated virus vectors transduce primary cells much less efficiently than immortalized cells. J. Virol. 69:1473-1479.

18. Heim, R., D. C. Prasher, and R. Y. Tsien. 1994. Wavelength mutations and posttranslational autooxidation of green fluorescent protein. Proc. Natl. Acad. Sci. USA 91:12501-12504.

19. Hoffman, M. A., and A. C. Palmenberg. 1995. Mutational analysis of the J-K stem-loop region of the encephalomyocarditis virus IRES. J. Virol. 69:43994406.

20. Jolly, D. 1994. Viral vector systems for gene therapy. Cancer Gene Ther. 1:55-64.

21. Kaplitt, M. G., P. Leone, R. J. Samulski, X. Xiao, D. W. Pfaff, K. L. O'Malley, and M. J. During. 1994. Long-term gene expression and phenotypic correction using adeno-associated virus vectors in the mammalian brain. Nat. Genet. 8:148-154.

22. Koenig, S., H. E. Gendelman, J. M. Orenstein, M. C. Dal Canto, G. H. Pezeshkpour, M. Yungbluth, F. Janotta, A. Aksamit, M. A. Martin, and A. S. Fauci. 1986. Detection of AIDS virus in macrophages in brain tissue from
AIDS patients with encephalopathy. Science 233:1089-1093.

23. Kubota, S., L. Duan, R. A. Furuta, M. Hatanaka, and R. J. Pomerantz. 1996 Nuclear preservation and cytoplasmic degradation of human immunodeficiency virus type 1 Rev protein. J. Virol. 70:1282-1287.

24. McLeod, G. X., J. M. McGrath, E. A. Ladd, and S. M. Hammer. 1992. Didanosine and zidovudine resistance patterns in clinical isolates of human immunodeficiency virus type 1 as determined by a replication endpoint concentration assay. Antimicrob. Agents Chemother. 36:920-925.

25. Miller, J. L., R. E. Donahue, S. E. Sellers, R. J. Samulski, N. S. Young, and A. W. Nienhuis. 1994. Recombinant AAV-mediated expression of a human gamma-globulin gene in human progenitor-derived erythroid cells. Proc. Natl. Acad. Sci. USA 91:10183-10187.

26. Muro-Cacho, C. A., R. J. Samulski, and D. Kaplan. Gene transfer in human lymphocytes using a vector based on AAV. 1992. J. Immunother. 11:231237.

27. Muzyczka, N. 1992. Use of adeno-associated virus as a general transduction vector for mammalian cells. Curr. Top. Microbiol. Immunol. 158:97-131.

28. Naldini, L., U. Blomer, P. Gallay, D. Ory, R. Mulligan, F. H. Gage, I. M Verma, and D. Trono. 1996. In vivo gene delivery and stable transduction of nondividing cells by a lentiviral vector. Science 272:263-267.

29. Pinkston, P., N. Pelletier, C. Arena, J. Schock, R. Garland, and R. M. Rose 1995. Quantitative culture of HIV-1 from bronchoalveolar lavage cells. Am. J. Respir. Crit. Care Med. 152:254-259.

30. Pomerantz, R. J., T. Seshamma, and D. Trono. 1992. Efficient replication of human immunodeficiency virus type 1 requires a threshold level of Rev: potential implications for latency. J. Virol. 66:1809-1813.

31. Pomerantz, R. J., D. Trono, M. B. Feinberg, and D. Baltimore. 1990. Cells nonproductively infected with HIV-1 exhibit an aberrant pattern of viral RNA expression: a molecular model for latency. Cell 61:1271-1276.

32. Pritchard, C. E., J. A. Grasby, F. Hamy, A. M. Zacharek, M. Singh, J. Karn, and M. J. Gait. 1994. Methylphosphonate mapping of phosphate contacts critical for RNA recognition by the human immunodeficiency virus tat and rev proteins. Nucleic Acids Res. 22:2591-2600.

33. Samulski, R. J., L. S. Chang, and T. Shenk. 1989. Helper-free stocks of recombinant adeno-associated viruses: normal integration does not require viral gene expression. J. Virol. 63:3822-3828.

34. Samulski, R. J., L. S. Chang, and T. Shenk. 1987. A recombinant plasmid from which an infectious adeno-associated virus genome can be excised in vitro and its use to study viral replication. J. Virol. 61:3096-3101.

35. Samulski, R. J., X. Zhu, X. Xiao, J. D. Brook, D. E. Housman, N. Epstein, and L. A. Hunter. 1991. Targeted integration of adeno-associated virus (AAV) into human chromosome 19. EMBO J. 10:3941-3950.

36. Schnittman, S. M., M. C. Psallidopoulos, H. C. Lane, L. Thompson, and C. A. Rosen. 1992. HIV regulatory proteins: potential targets for therapeutic intervention. AIDS Res. Hum. Retroviruses 8:175-181.

37. Vella, S. 1995. Clinical experience with saquinavir. AIDS 9:S21-S25.

38. Woffendin, C., Z. Y. Yang, Udaykumar, L. Xu, N. S. Yang, M. J. Sheehy, and G. J. Nabel. 1994. Nonviral and viral delivery of a human immunodeficiency virus protective gene into primary human T-cells. Proc. Natl. Acad. Sci. USA 91:11581-11585.

39. Wu, Y., L. Duan, M. Zhu, B. Hu, S. Kubota, O. Bagasra, and R. J. Pomerantz. 1996. Binding of intracellular anti-Rev single-chain variable fragments to different epitopes of human immunodeficiency virus type $1 \mathrm{Rev}$ : variations in viral inhibition. J. Virol. 70:3290-3297.

40. Xiao, X., W. deVlaminck, and J. Monahan. 1993. Adeno-associated virus (AAV) vectors for gene transfer. Adv. Drug Del. Rev. 12:201-215. 